\title{
Space Economy Grand Challenges
}

\author{
Martin Elvis* \\ Center for Astrophysics | Harvard and Smithsonian, Cambridge, MA, United States
}

Keywords: economy, technology, law, policy, design, ethics, arts

The space economy is changing fast. For decades, new civilian uses of space emerged at a stately pace-communications in the 1960s, TV broadcasts in the 1970s, and navigation in the 1980s. In strong contrast, new uses for space are now emerging rapidly and seemingly all at once. Companies are now working to make profits in many areas: earth observation, global high-speed internet, tourism, debris removal, research, manufacturing, construction, resource extraction, and, later, in settlement, threat mitigation, and security. In parallel with this, new regulatory regimes are being formed along with the development of legal frameworks for the use of space.

This emerging space economy presents a Grand Challenge for anyone who wants to keep pace and follow developments in a more nuanced way than merely scanning the headlines. There has been no primary journal in which all these aspects of the space economy have been brought together to allow these many areas of expertise to interact. Instead, the many papers published in these fields have been scattered over a wide variety of journals. This has led to a dispersed literature that makes it challenging to be aware of what has already been done and how current thinking is developing. In this way, it is not easy to establish a shared core of scholarship. Discussions among experts across disciplines is especially limited.

Space Economy has been founded to take up this grand challenge by providing a forum to promote discussion between all aspects of the space economy. The technological and mission design aspects of space are often considered in isolation. While it is true that initial feasibility is a purely technical issue, the goal of profitability ties the technological solutions inextricably to other areas. The immediate grand challenge for most companies is closing the business case with paying customers. Without this there will be no space economy. Many cases work well only if there is already a customer in space-a classic chicken-or-the-egg problem. Most obviously, then, business and economics are essential ingredients. But legal issues quickly arise. Those issues are then linked to questions of policy and governance. In these new and untried environments, they awaken new questions of ethics. As people in space become more common, expertise in architecture and design, as well as in sports and the arts, seem likely to become important. Space Economy aims to provide a platform upon which all these topics and their multi-directional interactions can be discussed both within individual papers and between them.

Space Economy will primarily publish original research articles. We plan to publish papers relating to all areas dealing with economic activities in space. These will mostly focus on short-range to medium-term issues. They may even cover issues relevant to prospects that will not be 
realized for a century. We prefer work with an emphasis on concrete thinking rather than abstract speculation. Space Economy will also publish subject reviews, catalogs, book reviews, and occasional opinion pieces if they are based on scholarship. Some papers may be cross listed with other sections in Frontiers in Space Technologies; for example, space debris articles may be appropriate for Space Economy as well as for Debris.

Our aim regarding Space Economy is to foster a community of scholarship around the new space economy. The Open Access model of the Frontiers Community Journals encourages inclusiveness-a necessity for the flourishing of such a community. We hope you agree.

\section{AUTHOR CONTRIBUTIONS}

The author confirms being the sole contributor of this work and has approved it for publication.

Conflict of Interest: The author declares that the research was conducted in the absence of any commercial or financial relationships that could be construed as a potential conflict of interest.

Copyright (c) 2020 Elvis. This is an open-access article distributed under the terms of the Creative Commons Attribution License (CC BY). The use, distribution or reproduction in other forums is permitted, provided the original author(s) and the copyright owner(s) are credited and that the original publication in this journal is cited, in accordance with accepted academic practice. No use, distribution or reproduction is permitted which does not comply with these terms. 